\title{
Micro bubble dynamics in DNA solutions
}

\author{
Peigang Deng ${ }^{1}$, Yi-Kuen Lee ${ }^{1}$ and Ping Cheng ${ }^{2}$ \\ ${ }^{1}$ Mechanical Engineering Department, Hong Kong University of Science and Technology, \\ Clear Water Bay, Kowloon, Hong Kong, People's Republic of China \\ ${ }^{2}$ School of Mechanical and Power Engineering, Shanghai Jiaotong University, \\ Shanghai 200030, People's Republic of China \\ E-mail:meyklee@ust.hk
}

Received 4 October 2003

Published 17 March 2004

Online at stacks.iop.org/JMM/14/693 (DOI: 10.1088/0960-1317/14/5/006)

\begin{abstract}
Micro bubble generation and its subsequent dynamic behavior in single-stranded DNA (ssDNA) solutions are presented in this paper. A micro vapor bubble was generated in ssDNA using a micro bubble actuator, which is capable of producing periodic and stable single vapor bubbles under pulse heating. The growth and collapse of the micro vapor bubble were visualized by a high-speed CCD camera, and the bubble dynamics was investigated at different ssDNA concentrations and under various pulse widths. It was observed that an increase in the ssDNA concentration led to an increase of the electric power required for incipient bubble nucleation. Based on thermodynamics considerations and a simple model for nucleus formation in ssDNA solution, an analysis of bubble nucleation work was carried out and the results are consistent with experimental data. It is found that the bubble dynamics in ssDNA solutions is different from that in DI water, and an obvious retardation effect on the motion of the micro bubble was observed at high ssDNA concentrations. Based on Zimm's model, the effect of ssDNA macromolecules on the total viscosity of the solution is revealed. The present study indicates that polymer properties can significantly influence bubble nucleation and the subsequent evolution of bubble dynamics, owing to hydrodynamic intermolecular interactions of polymer macromolecules.
\end{abstract}

$\begin{array}{ll}\text { Nomenclature } \\ C & \begin{array}{l}\text { concentration of the polymer } \\ \text { current run through the micro heater }\end{array} \\ K_{\mathrm{B}} & \text { Boltzmann constant } \\ L & \text { equivalent size of the micro heater } \\ L_{\mathrm{c}} & \text { contour length } \\ M & \text { molecular weight } \\ n & \text { number of the vapor molecules in a liquid-vapor } \\ N_{\mathrm{A}} & \text { nucleus system } \\ P_{\mathrm{h}} & \text { Avogadro's number } \\ P_{1} & \text { input power to the micro heater } \\ P_{\mathrm{v}} & \begin{array}{l}\text { pressure of the vapor nucleus } \\ \text { pressure at a liquid-vapor equilibrium condition }\end{array} \\ P_{\mathrm{equ}} & \begin{array}{l}\text { radius of the spherical nucleus } \\ r\end{array} \\ r_{\mathrm{cr}} & \begin{array}{l}\text { critical radius of the spherical nucleus, where the } \\ \text { bubble nucleation work reaches its maximum }\end{array}\end{array}$

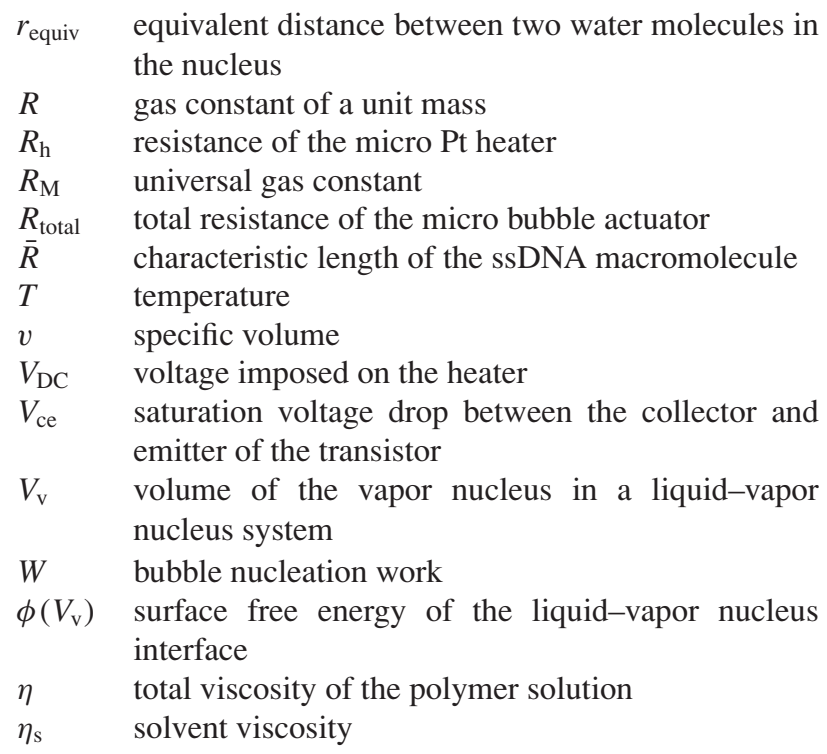


$[\eta] \quad$ intrinsic viscosity

$\mu_{1} \quad$ chemical potential of the molecules in liquid phase

$\mu_{\mathrm{v}} \quad$ chemical potential of the molecules in vapor nucleus phase

$\mu_{\mathrm{v}, \mathrm{um}} \quad$ chemical potential of unit mass vapor

$\bar{\mu}_{\mathrm{v}, \mathrm{um}}$ equilibrium chemical potential of unit mass vapor

$\mu_{1, \text { um }} \quad$ chemical potential of unit mass liquid

$\bar{\mu}_{1, \text { um }}$ equilibrium chemical potential of unit mass liquid

$\sigma \quad$ surface tension

$\tau \quad$ relaxation time

$\tau_{1} \quad$ heating pulse width

$\tau_{2} \quad$ waiting pulse width

$\xi_{\mathrm{p}} \quad$ DNA persistence length

\section{Introduction}

Recently, there has been a great deal of interest in using micro heaters to generate micro vapor bubbles for applications in MEMS devices. Utilizing the expansion work of bubble generation, these micro bubble actuators have been applied to inkjet printheads, micropumps, micromixers, etc $[1,2]$. Theoretical and experimental researches on rapid boiling and bubble generation from a micro heater have been carried out by a number of investigators [3-5]. It can be concluded from these studies that homogeneous boiling is the most likely mechanism for micro bubble generation on the pulse-heated micro heater. Because of the micro size heater and the short duration of pulse heating, the order of magnitude of heat flux in these applications is usually as high as $\mathrm{MW} \mathrm{m}^{-2}$, which is quite different from those in the macro scale boiling process. Recently, Deng et al [6, 7] have investigated the possibility of using a micro vapor bubble as a thermal actuator to enhance the mixing process in a microsystem. It is shown that an asymmetric bubble growth-collapse cycle can introduce effective flow perturbations in the bulk fluid.

In recent years, micro bubble technology has also been applied to the biomedical field. A micro bubble jet printing device was used to eject DNA segments (presynthesized oligonucleotides) onto a glass surface for DNA micro-array [8]. Some physical properties of the DNA solution, such as volatility, solubility, wettability, viscosity and surface tension, were optimized by chemical additives in order to reduce possible high-temperature and hydrodynamic damages to DNAs, which were induced by the transient vapor bubble formation process.

Generally speaking, vapor bubble generation in polymers [9-11] is more complicated than the corresponding problems in a pure substance [12] because the former involves phase change in a multi-component fluid. In the study of the cavitation problem in polymer solutions, an initial small noncondensable gas bubble was presumed to exist in the solution, and the adiabatic expansion-compression oscillation of the gas bubble was owing to pressure fluctuations in the fluid. The influence of different polymer properties on the damping effect on the bubble radius versus time has been investigated numerically and experimentally in these studies [9-11].

In this paper, micro vapor bubble generation in singlestranded DNA (ssDNA) solutions is investigated using a micro bubble actuator that is capable of producing periodic and stable single vapor bubbles under pulse heating. The growth and

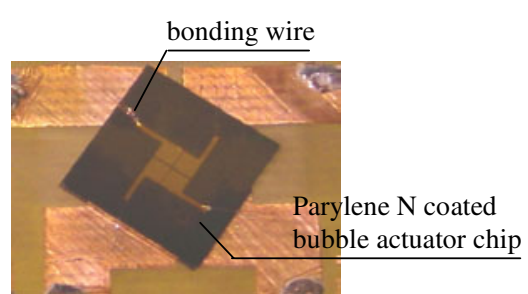

(a)

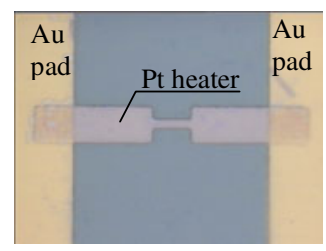

(b)

Figure 1. Picture of the micro vapor bubble actuator: $(a)$ packaged micro bubble actuator, $(b)$ non-uniform width micro heater.

collapse of a micro vapor bubble were observed using a highspeed CCD camera. The effects of ssDNA concentration on the power input required for incipient bubble generation and the bubble dynamic behavior are investigated. The reason for choosing the ssDNA solution as the test fluid is because DNA hybridization between ssDNAs (with one acting as DNA probe and the other as target DNA) is the operational principle for many DNA-based biosensors. Based on thermodynamics considerations and a simple model for nucleus formation in ssDNA solution, an analysis is carried out to evaluate the variation of the bubble nucleation work with the addition of ssDNA macromolecules in the aqueous solution. The results of this investigation give better understanding of the micro bubble generation process in DNA, which has been extensively employed in DNA sample transport based on micro thermal ink jet printer technology [8].

\section{Micro bubble actuator}

\subsection{Design and fabrication of the micro heater}

Figure 1 $(a)$ is an overall picture of the packaged micro bubble actuator chip, which consists of a specially designed micro heater $\left(10 \times 2 \mu \mathrm{m}^{2}\right)$ and the electric pad. Figure $1(b)$ is a close-up view of the micro heater under a microscope. The specially designed micro heater differs from a conventional strip heater in that it is not uniform in width and has a slim part in the middle section of the heater. The non-uniformwidth design of the micro heater ensures the controllability and reliability of the bubble generation process, which can produce a single vapor bubble exactly on the slim part of the heater under pulse heating. The detailed explanation and analysis of this kind of micro bubble actuator is given in [6].

The length and width of the micro heater were $50 \mu \mathrm{m}$ and $10 \mu \mathrm{m}$, respectively, while the length and width of the slim part were $10 \mu \mathrm{m}$ and $2 \mu \mathrm{m}$, respectively. Pt was chosen as the material for the heater and Au for the electric pad. The fabrication process of the micro heater followed the conventional MEMS technology. A n-type, double-sided polished, and $\langle 100\rangle$ oriented Silicon wafer (4-inch in diameter 
and $400 \mu \mathrm{m}$ in thickness) was used as the substrate. The wafer was coated with $0.5 \mu \mathrm{m}$ silicon oxide and $1.5 \mu \mathrm{m}$ low stress silicon nitride on both sides of the wafer by wet thermal oxidation and low-pressure chemical vapor deposition (LPCVD) processes, respectively. A $0.01 \mu \mathrm{m}$ thick Ti film and $0.15 \mu \mathrm{m}$ thick Pt film were sputtered on the wafer and patterned using the lift-off method. Finally, a $0.2 \mu$ m thick Au film was sputtered after a $0.01 \mu \mathrm{m}$ thick TiW sputtering, and patterned also by the lift-off method. Both the length and width of the Au pad were $300 \mu \mathrm{m}$. After the micro fabrication process, the micro bubble actuator chip was mounted on a printed circuit board (PCB), and the electric pad on the chip was wire bonded to the PCB (figure $1(a)$ ).

\subsection{Surface treatment of the bubble actuator chip}

To avoid direct contact of the DNA solution with the heater and the electric pad, an isolation layer on the micro bubble actuator chip is needed. For this purpose, a gas-phase vacuum deposited parylene conformal coating was adopted [13]. As a chemical-inert polymer, parylene can provide a biocompatible and dielectric isolation layer, and the hydrophobic and lubricous nature of parylene coating can minimize residual fluid build-up. Furthermore, the parylene coating is pinhole free and can be effective in an extremely thin layer, which is of great importance in view of the heat transfer efficiency from the heater underneath the parylene to the liquid on top of it.

In this micro bubble actuator chip, the high-quality parylene $\mathrm{N}$ (diX-N ${ }^{\circledR}$, Daisankasei Co., Ltd, Chiba, Japan) was adopted as the deposition material because of its high degree of thermal stability (melting point $420^{\circ} \mathrm{C}$ ) and high dielectric strength. The deposition process was carried out on a SCS parylene deposition system (PDS-2010) after the packaging of the micro bubble actuator chip. The operation conditions were: furnace temperature at $650{ }^{\circ} \mathrm{C}$, vaporizer temperature at $160{ }^{\circ} \mathrm{C}$ and a vacuum of 60 mTorr. Since the cure cycle was automatic with parylene and occurred before deposition, no testing or examination is required to confirm that parylene was fully cured. In consideration of the heat transfer efficiency, the thickness of the deposited parylene thin film was chosen to be about $60 \mathrm{~nm}$ (measured on Tencor P-10 Surface Profiler). Figure 1 shows the micro bubble actuator chip after parylene deposition.

\section{Experimental set-up and DNA sample}

\subsection{Experimental set-up}

In this experiment, the test liquid (ssDNA solutions) was put onto the parylene surface of the micro bubble actuator chip by a pipette, isolated with the micro heater by the parylene thin film. Figure 2(a) is a schematic diagram of the experimental set-up, where a high-speed CCD camera (MotionScope, PCI 8000s, Redlake Inc., USA) was connected to a microscope, which was synchronized with the heating process. The CCD had a maximum speed of 2000 frames per second. The heating pulse was controlled by a switching circuit, which could provide pulse width as short as several microseconds. The control circuit for the heating pulse generator is schematically shown in figure 2(b). A programmable $8255 \mathrm{I} / \mathrm{O}$ card was employed to generate a pulse train, which was sent to the base terminal of

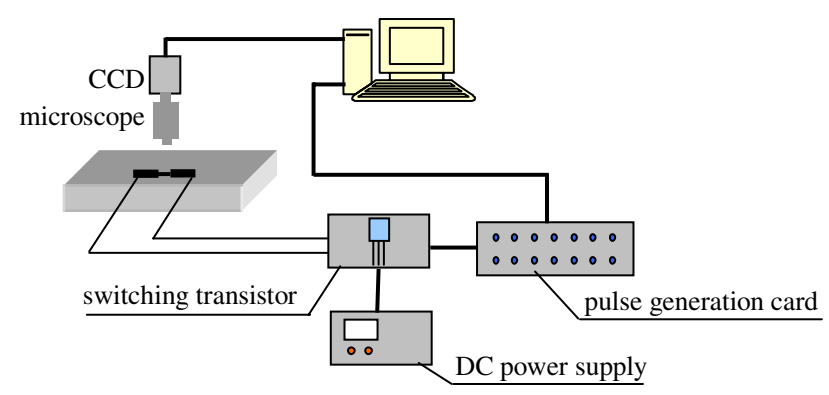

(a)

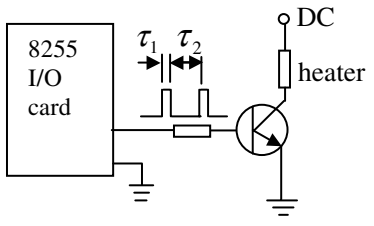

(b)

Figure 2. (a) Schematic diagram of experimental set-up and (b) control circuit of the micro heater.

a high gain transistor (ZTX689B, Zetex) to control the on/off heating process. The signals were also sent to the CCD camera to trigger the recording process. The switching time of the transistor was at nanosecond level according to the datasheet. A DC power supply (model DF1731SB by Goldsource) and the micro heater were connected to the collector terminal of the transistor, and the emitter terminal was grounded. The pulse width, $\tau_{1}$ in figure $2(b)$, varied from several microseconds to milliseconds. To avoid the overlapping of bubbles, a waiting pause $\tau_{2}$ of $1 \mathrm{~s}$ was inserted between two consecutive heating pulses. Note that the CCD camera recording process was synchronized with the heating process.

\subsection{DNA sample preparation}

A 60-base synthetic oligonucleotide (5'-ACTAG GTCAC ACTAG GTCAC ACTAG GTCAC CACTG GATCA CACTG GATCA CACTG GATCA-3', Invitrogen, Inc., USA) was used in this experiment. The lyophilized ssDNA was first centrifuged and dissolved completely in $1 \times$ TE buffer (10 mM Tris-HCl, 1mM EDTA, pH 7.4). It was then diluted with distilled water into four concentrations: $0.1 \mu \mathrm{g} \mu \mathrm{l}^{-1}$, $1 \mu \mathrm{g} \mu \mathrm{l}^{-1}, 6.4 \mu \mathrm{g} \mu \mathrm{l}^{-1}$ and $10 \mu \mathrm{g} \mu \mathrm{l}^{-1}$.

\section{Bubble nucleation in DNA solutions}

\subsection{Experimental observation of micro bubble dynamics}

In this section, we discuss the experimental study of bubble generation and collapse process in ssDNA solutions heated by a micro heater, with particular emphasis on bubble dynamics under various ssDNA concentrations and different heating pulse widths.

4.1.1. Concentration effects on bubble dynamics. After loading the ssDNA sample onto the micro bubble actuator chip, a typical $1.6 \mathrm{~ms}$-width heating pulse was imposed on 


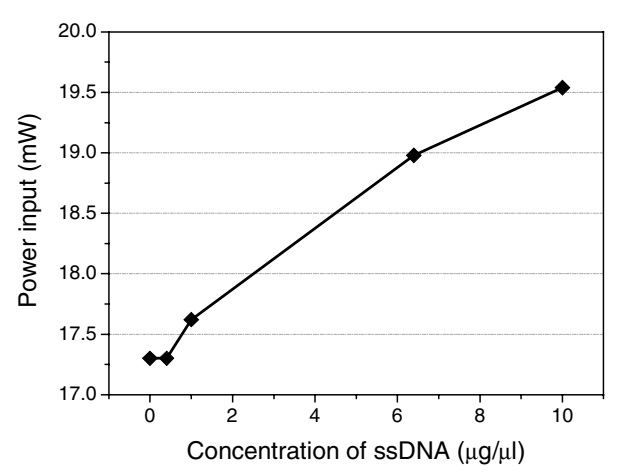

Figure 3. Input power for incipient bubble generation in ssDNA solution at various concentrations.

the micro heater. The current run through the micro heater, $I$, can be expressed as

$$
I=V_{\mathrm{DC}}-V_{\mathrm{ce}} / R_{\mathrm{total}}
$$

where $V_{\mathrm{DC}}$ is the imposed voltage (figure $2(b)$ ), $V_{\text {ce }}$ is the saturation voltage drop between the collector and emitter of the transistor and $R_{\text {total }}$ is the total resistance of the micro bubble actuator, which includes the resistance of the micro heater $R_{\mathrm{h}}$, the resistance of the Au electric pad and the contact resistance. Note that $R_{\text {total }}$ can be measured directly using a multimeter, and $R_{\mathrm{h}}$ can be calculated based on the sheet resistance of the Ti/Pt film (1.64 $\Omega / \square$ for the present case) and the size of the heater. Thus, the input power to the micro heater, $P_{\mathrm{h}}$, can be expressed as

$$
P_{\mathrm{h}}=I^{2} R_{\mathrm{h}}
$$

where $R_{\mathrm{h}}$ is the resistance of the micro heater. It is worthwhile to point out that the input power to the micro heater obtained by equation (2) is actually the one at the beginning of the pulse heating process, and it will change with time because the resistance of the micro heater will change as its temperature increases. In the experiment, the input power was gradually increased until boiling was observed in the DNA solution. It was found that with the increase of the DNA concentration, the input power required for incipient bubble generation was also increased. As shown in figure 3, the input power for bubble generation in $0.4 \mu \mathrm{g} \mu 1^{-1}$ ssDNA was equal to that in DI water $(17.3 \mathrm{~mW})$, and it was increased by $2 \%$ in $1 \mu \mathrm{g} \mu \mathrm{l}^{-1}$ ssDNA with respect to that for DI water. In $6.4 \mu \mathrm{g} \mu \mathrm{l}^{-1}$ ssDNA, the input power was increased about $10 \%$ while in $10 \mu \mathrm{g} \mu \mathrm{l}^{-1}$ ssDNA, it was increased by $13 \%$. Obviously, the nucleus formation process was affected by the existence of ssDNA macromolecules in the solution. With the increase of the DNA concentration, a larger power input was required for the incipient bubble nucleation. This phenomenon will be further analyzed in the next section based on classical thermodynamic theory on nucleation work and a simple model for nucleus formation in ssDNA solution.

Figure 4 shows a series of snapshots taken by a highspeed CCD camera at different times during the micro bubble growth and collapse process in $1 \mu \mathrm{g} \mu \mathrm{l}^{-1}$ ssDNA solution. From the video, we can see that a single micro bubble was generated precisely on the slim part of the non-uniform heater, and then it grew rapidly until reaching its maximum size. It should be noted that due to the limitation of the CCD

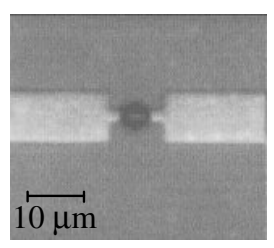

$0.5 \mathrm{~ms}$

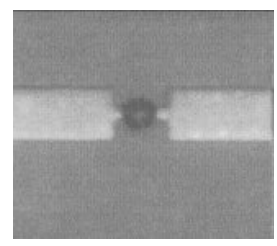

$1 \mathrm{~ms}$

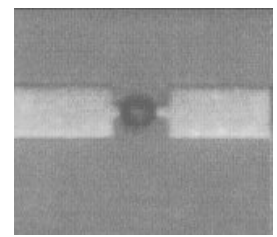

$1.5 \mathrm{~ms}$

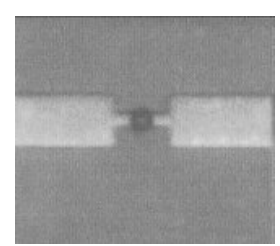

$2 \mathrm{~ms}$

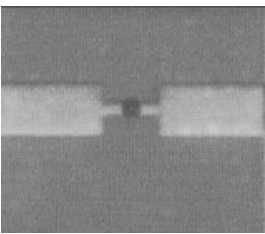

$50 \mathrm{~ms}$

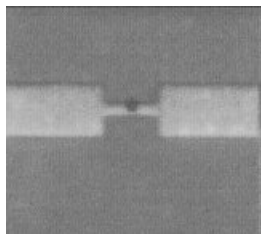

$100 \mathrm{~ms}$
Figure 4. Micro single bubble observed in $1 \mu \mathrm{g} \mu \mathrm{l}^{-1}$ ssDNA solution. Pulse heating width: $1.6 \mathrm{~ms}$, and pulse heating power: $17.62 \mathrm{~mW}$

recording speed of 2000 frames per second (1 frame per $0.5 \mathrm{~ms}$ ), some information was lost during this rapidly growing process. After reaching its maximum size, the bubble began to collapse on the surface of the micro heater. No bubble departure was observed in the repeated tests in this experiment because of the short heating period. It should be noted that the generation of a single bubble in the present experiment can be attributed to a highly localized near-homogeneous boiling mechanism. For a detailed description of this kind of boiling, the reader is referred to our previous studies $[6,7]$.

Although single bubble was also observed in three other concentrations $\left(0.4 \mu \mathrm{g} \mu \mathrm{l}^{-1}, 6.4 \mu \mathrm{g} \mu \mathrm{l}^{-1}\right.$ and $\left.10 \mu \mathrm{g} \mu \mathrm{l}^{-1}\right)$ of the ssDNA solution, the bubble growth/collapse processes were quite different from one another. In order to characterize single bubble dynamics at each ssDNA concentration quantitatively, the bubble diameter in each frame of the digitalized videos was measured using a Matlab program. It is pertinent to note that the error of the present bubble size measurement method stemmed mainly from the difficulty of identifying the borderline of the bubble geometry accurately. An absolute error of $\pm 0.2 \mu \mathrm{m}$ was found based on repeated measurements for the same bubble picture.

Figure 5(a) shows the bubble diameter versus time in ssDNA solutions with different concentrations. In this figure, time is normalized by the heating pulse width $(1.6 \mathrm{~ms})$ and bubble diameter is normalized by the equivalent size of the micro heater $L$, which is defined as the square root of the area of the slim part of the heater, namely, $L=\sqrt{10 \times 2}=4.5 \mu \mathrm{m}$. As clearly illustrated in figure 5(a), the overall bubble lifetime in ssDNA solutions decreased distinctly with the increase of ssDNA concentration. For example, the bubble lifetime in $10 \mu \mathrm{g} \mu \mathrm{l}^{-1}$ ssDNA was only $1 / 7$ of that in $0.4 \mu \mathrm{g} \mu \mathrm{l}^{-1}$. For comparison, the bubble growth/collapse in DI water is also presented in the same graph, which had the longest lifetime. 


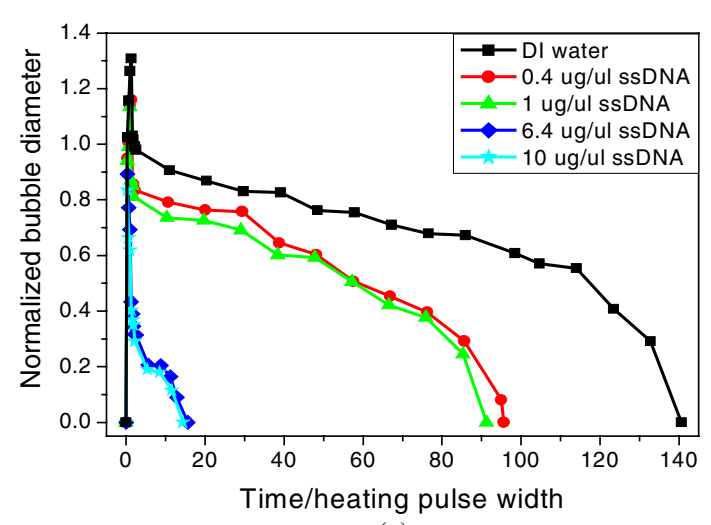

(a)

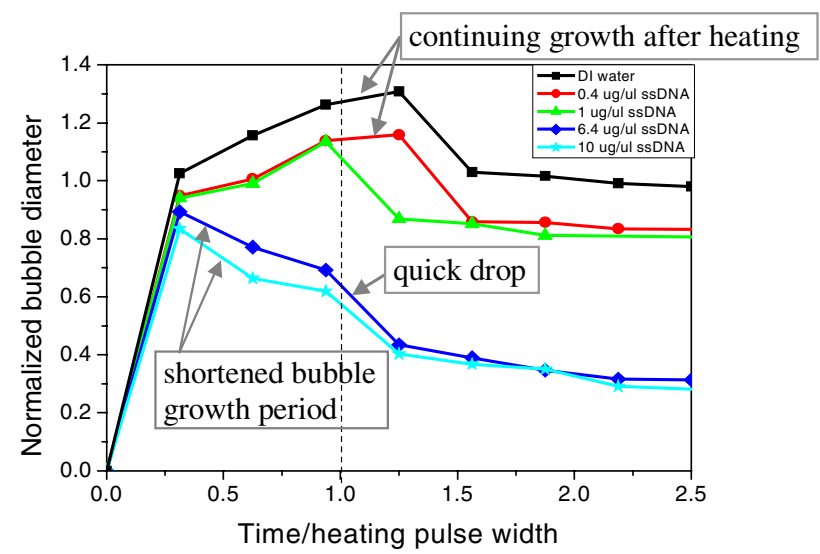

(b)

Figure 5. Micro bubble diameter versus time in ssDNA solutions: (a) bubble diameter-time history, (b) close-up view of the bubble growth process. The heating pulse width is $1.6 \mathrm{~ms}$ and the heating power for each case is shown in figure 3 .

For all testing concentrations, the bubble growth period (which was comparable to the heating pulse width) was much shorter than the bubble collapse process.

Since the bubble growth process was much shorter than the collapse process, a close-up view of the bubble growth process is given in figure $5(b)$. It is shown that the collapse process started with a sudden shrink in diameter, followed by a slow and gradual collapsing process until the bubble disappeared. In addition to the bubble lifetime, the bubble growth process was also highly affected by the DNA concentration. As shown in figure 5(b), the bubble growth process was obviously shortened with the increase of the DNA concentration. For the cases of DI water and $0.4 \mu \mathrm{g} \mu \mathrm{l}^{-1}$ ssDNA solution, the maximum-size bubbles were both recorded at $2 \mathrm{~ms}$ after the beginning of the heating process, implying that the bubble kept on growing even though the heating pulse stopped at $1.6 \mathrm{~ms}$. This behavior is very similar to our previous studies $[6,7]$. For the case of $1 \mu \mathrm{g} \mu \mathrm{l}^{-1}$, the maximum-size bubble was recorded at $1.5 \mathrm{~ms}$, implying that the bubble stopped growing at almost the end of the heating pulse. For high concentrations, $6.4 \mu \mathrm{g} \mu \mathrm{l}^{-1}$ and $10 \mu \mathrm{g} \mu \mathrm{l}^{-1}$, however, the maximum-size bubbles were both recorded at $0.5 \mathrm{~ms}$, indicating that the bubble growing process was much shorter than that at moderate concentrations, and the bubble stopped growing even though heating from the heater was still going on. Figure $5(b)$ also shows that the

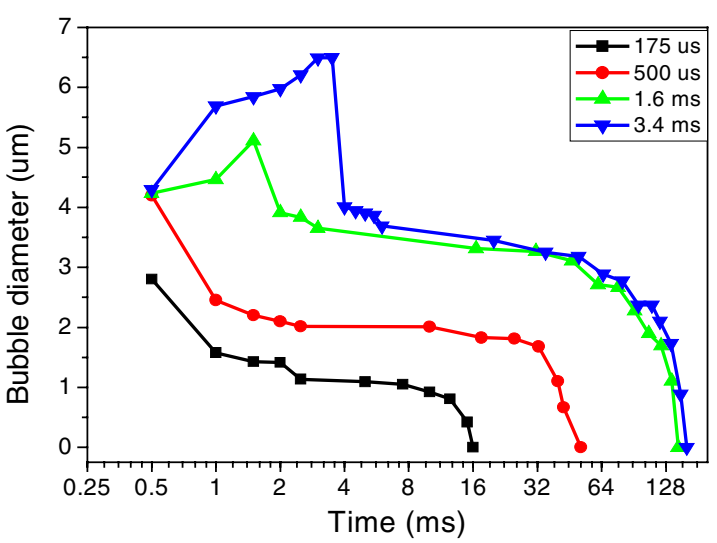

Figure 6. Semi-logarithmic plot of bubble diameter versus time in $1 \mathrm{ug} \mathrm{ul}^{-1}$ ssDNA solution under different heating pulse widths.

maximum bubble diameter decreases with the increase of the ssDNA concentration. However, it should be reminded that the maximum diameter of the bubble recorded might not be the real size. This is owing to the limitation of the CCD camera recording speed. It is believed that the maximum bubble diameter is of the same order of magnitude with the equivalent size of the micro heater, because the superheated area in the liquid is of comparable size with the heater area in a micro-scale rapid heating process [6].

4.1.2. Bubble dynamics under various heating pulse widths. Using $1 \mu \mathrm{g} \mu \mathrm{l}^{-1}$ ssDNA test sample, bubble dynamics was studied under different heating pulse widths of the micro bubble actuator, ranging from $175 \mu \mathrm{s}$ to $3.4 \mathrm{~ms}$. For all cases, input powers were fixed at $17.62 \mathrm{~mW}$, the power required for incipient bubble generation in $1 \mu \mathrm{g} \mu \mathrm{l}^{-1}$ ssDNA with a $1.6 \mathrm{~ms}$-wide heating pulse. Figure 6 is a semi-logarithmic plot of bubble diameter versus time under different heating widths. As shown in the figure, the bubble growth period is very close to the pulse width for each case, which is quite different from that at high DNA concentrations as shown in figure $5(b)$. The bubble collapse process shortens with the decrease of the heating width due to the shrinking of the superheated region in the liquid [6]. No bubble departure was observed for all cases because of the short heating period.

\subsection{Analysis on bubble dynamics}

From the aforementioned experimental observation of bubble dynamics in DI water and in DNA solutions with various concentrations, it is quite clear that polymer properties have significant influence on bubble nucleation and behavior. From our previous study on bubble dynamics in two common DNA buffers $(20 \mathrm{mM}$ Tris-HC1 and $1 \times$ TAE), both the bubble diameter-time history and the bubble lifetime in the DNA buffers are very similar to those in DI water [7]. Furthermore, the input power for incipient bubble generation in the two DNA buffers is the same as that in DI water. This implies that the distinctive bubble dynamics observed in the present experiments are mainly due to the existence of the ssDNA macromolecules, rather than the solvent molecules. In the following, we shall discuss some bubble dynamic 


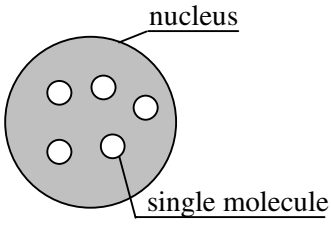

superheated liquid

(a)

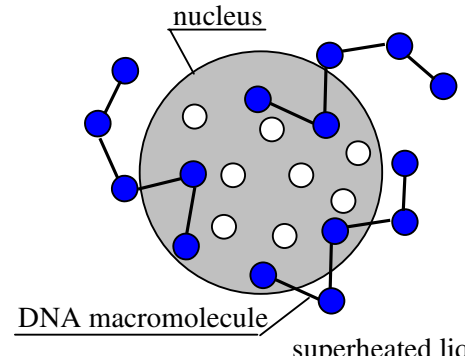

(b)

Figure 7. Schematic diagram of nucleus formation in liquid: (a) nucleation in single-component liquid, $(b)$ nucleation in multi-component liquid, ssDNA is modeled as a freely joint chain (FJC).

behaviors that are affected by the existence of the ssDNA macromolecules: input power for incipient bubble nucleation, bubble growth process, maximum size of bubble and the bubble collapse process.

4.2.1. Bubble nucleation work. In general, the formation of bubbles in phase change situations involves an initiation stage called nucleation, where a nanoscale nucleus of vapor phase is formed in the metastable (supersaturated or superheated) bulk liquid phase. From molecule kinetics [14], random fluctuations of liquid molecules will cause micro scale density fluctuations, and the fluctuations will become drastic in a superheated liquid. Thus, the low-density region, created in bulk liquid that exhibits similar thermodynamic properties to a vapor bubble, can be considered as nucleus. Basically, the formation of a nucleus involves overcoming an energy barrier resulting from the change of a pertinent thermodynamic potential accompanying the nucleus formation. This energy barrier is the nucleation work. In the following, we will first analyze the bubble nucleation work in a pure substance based on classical thermodynamics, and then establish a model to describe the nucleus formation in ssDNA solution.

For simplicity, we consider homogeneous bubble nucleation in single-component systems that are in a state away from the critical point. Suppose that a nucleus forms locally as a density fluctuation in a spatial region occupied by $n$ molecules, as schematically illustrated in figure 7(a). The nucleation work can be expressed as the change of the system Gibbs free energy after and before the bubble nucleation, which reads [15-17]:

$$
W=n\left(\mu_{\mathrm{v}}-\mu_{1}\right)-\left(P_{\mathrm{v}}-P_{1}\right) V_{\mathrm{v}}+\phi\left(V_{\mathrm{v}}\right) .
$$

Equation (3) indicates that the nucleation work can be attributed to three effects: the first term is the variation of the chemical potential of the molecules in the volume of the nucleus when changing from the liquid phase to vapor phase. The second one is the pressure difference between the vapor nucleus and the liquid, which is the volumetric free energy. The third one is the additional surface free energy accompanying the appearance of the liquid-vapor nucleus interface.

Let us examine the chemical potential difference first. For simplicity, if we assume that the vapor phase is ideal and the liquid is incompressible, the following expressions can be obtained by integrating the Gibbs-Duhen equation to a liquidvapor equilibrium pressure $P_{\text {equ }}$ :

$$
\begin{gathered}
\mu_{\mathrm{v}, \mathrm{um}}-\bar{\mu}_{\mathrm{v}, \mathrm{um}}=R T \ln \left(\frac{P_{\mathrm{v}}}{P_{\text {equ }}}\right) \\
\mu_{1, \mathrm{um}}-\bar{\mu}_{1, \mathrm{um}}=v\left[P_{1}-P_{\text {equ }}\right] .
\end{gathered}
$$

Subtracting equation (5) from equation (4) and noting that $\bar{\mu}_{\mathrm{v}, \mathrm{um}}=\bar{\mu}_{1, \mathrm{um}}$, the chemical potential difference per unit mass is

$$
\mu_{\mathrm{v}, \mathrm{um}}-\mu_{1, \mathrm{um}}=R T \ln \left(\frac{P_{\mathrm{v}}}{P_{\text {equ }}}\right)-v\left[P_{1}-P_{\text {equ }}\right] .
$$

The above equation can be further simplified if we consider the relative magnitudes of the two terms on the right-hand side of the equation. Since the second term on the right-hand side of the above equation is much smaller than the first term, equation (6) can be reduced to

$$
\mu_{\mathrm{v}}-\mu_{1}=K_{\mathrm{B}} T \ln \left(\frac{P_{\mathrm{v}}}{P_{\text {equ }}}\right)
$$

where $K_{\mathrm{B}}=R_{\mathrm{M}} / N_{\mathrm{A}}=M R / N_{\mathrm{A}}$. An expression similar to equation (7) can also be found in [18], by replacing $\mu_{1}$ with the equal chemical potential of the vapor bubble being in unstable equilibrium state.

For a spherical nucleus with radius $r$, the surface free energy $\phi\left(V_{\mathrm{v}}\right)$ can be expressed as a function of the radius of the nucleus:

$$
\phi\left(V_{\mathrm{v}}\right)=4 \pi r^{2} \sigma .
$$

Treating the vapor phase as an ideal gas, the state equation gives

$$
P_{\mathrm{v}} V_{\mathrm{v}}=n K_{\mathrm{B}} T .
$$

Inserting equations (7)-(9) into equation (3), the nucleation work is

$$
W=4 \pi r^{2} \sigma-\frac{4 \pi r^{3}}{3}\left(P_{\mathrm{v}}-P_{1}\right)+\frac{4 \pi r^{3}}{3} P_{\mathrm{v}} \ln \left(\frac{P_{\mathrm{v}}}{P_{\text {equ }}}\right) .
$$

Note that it is difficult to find $P_{\mathrm{v}}$ because the vapor nucleus actually is not in equilibrium with liquid. However, if a mechanical equilibrium at the liquid-vapor interface is assumed [19], then, $P_{\mathrm{v}}$ is given by the Young-Laplace formula

$$
P_{\mathrm{v}}-P_{1}=\frac{2 \sigma}{r}
$$

We can examine the effects of various factors on the nucleation work based on equations (10) and (11). Figure 8 shows the calculated result of $W$ for bubble nucleation in water as a function of the nucleus radius $r$ at the nucleation temperature of $210{ }^{\circ} \mathrm{C}$, with $P_{1}$ at the ambient pressure of $1.01 \times 10^{5} \mathrm{~Pa}$ and $P_{\text {equ }}$ being the saturated vapor pressure corresponding to the nucleation temperature. An equivalent distance between two adjacent water molecules in the 


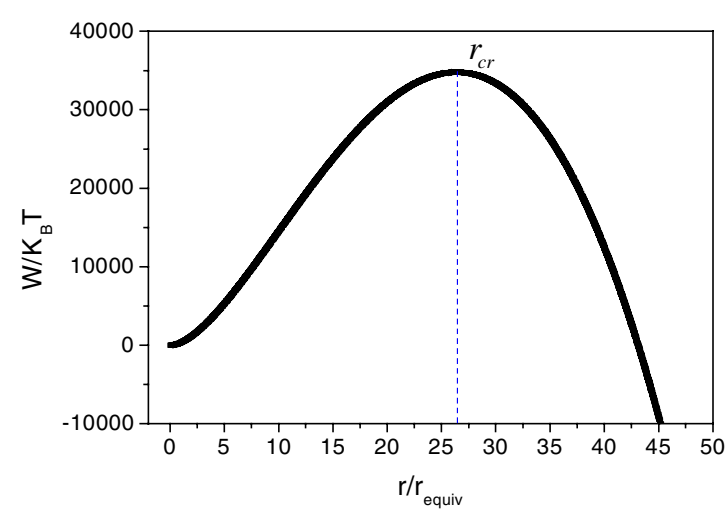

Figure 8. Nucleation work versus radius of the nucleus at a nucleation temperature of $210^{\circ} \mathrm{C}$.

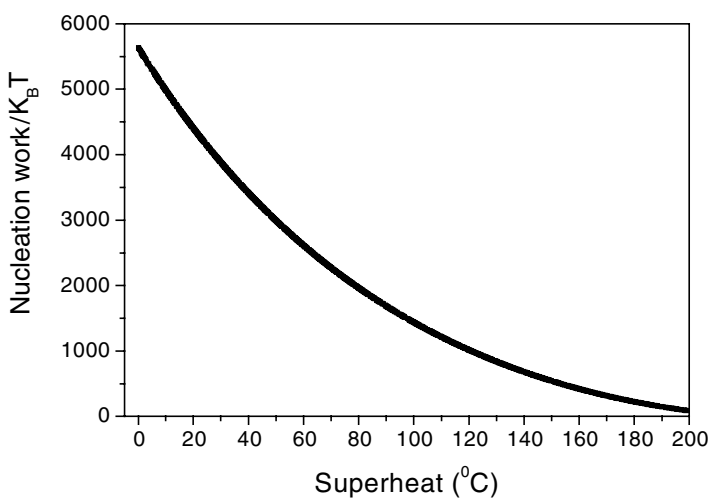

Figure 9. Nucleation work versus degree of superheat at $r=30^{\circ} \mathrm{A}$.

nucleus $r_{\text {equiv }}=15^{\circ} \mathrm{A}$ was chosen to normalize the nucleus radius $r$, and the nucleation work was normalized by $K_{\mathrm{B}} T$. As shown in figure 8, the nucleation work at first increases with increasing radius, passing through a maximum and then decreases. In equation (10), for small $r\left(r<r_{\mathrm{cr}}\right)$, the surface free energy grows faster $\left(r^{2}\right)$ than the volumetric free energy decreases $\left(r^{3}\right)$, so $W$ increases with $r$. On the other hand, when $r$ is larger than a critical value $r_{\mathrm{cr}}$, the volumetric energy term decreases faster than the growth of the surface term, so $W$ decreases with $r$. At the critical radius (indicated as $r_{\mathrm{cr}}$ in the figure) where $\mathrm{d} W / \mathrm{d} r=0$ and $\mathrm{d}^{2} W / \mathrm{d} r^{2}<0$, it implies that a metastable equilibrium is established at that point between the two phases. It is pertinent to note that for most cases, bubble nucleation occurs at $r<r_{\mathrm{cr}}[14,18]$; thereby a larger nucleation work is needed for a larger nucleus formation.

Figure 9 shows the variation of nucleation work for water as a function of superheat (with the boiling temperature at $100{ }^{\circ} \mathrm{C}$ ) at the nucleus radius $r=30{ }^{\circ} \mathrm{A}$, with $P_{1}=1.01 \times$ $10^{5} \mathrm{~Pa}$ and $P_{\text {equ }}$ being the saturated vapor pressure corresponding to its nucleation temperature. It is shown that the nucleation work decreases quickly with the increasing superheat of the water. This is because the surface energy term in equation (10) decreases with the increasing temperature due to the decrease of the surface tension.

With the above information, we now establish a simple model for nucleus formation in DNA solution. It can be speculated that the existence of the ssDNA macromolecules in the aqueous solution would make the bubble nucleation process different from that in pure water. Firstly, as mentioned earlier in this section, fluctuations of liquid molecules will facilitate the nucleus formation. The appearance of ssDNA macromolecules in the solution, however, will retard the fluctuation of the liquid molecules through hydrodynamic intermolecular interactions, especially at high concentrations. Secondly, since the ssDNA macromolecules can be modeled as freely-jointed-chains [20] as shown in figure $7(b)$, these macromolecules will displace the water molecules that previously occupied the space in pure water. On the other hand, the nucleus must contain enough water molecules to ensure that the statistical thermodynamic properties of the nucleus, say density, are close to that of a vapor phase. Thus, with the increase of the DNA concentration, a larger nucleus is expected in order to include enough water molecules. According to figure 8, a larger nucleus implies that a larger nucleation work is required for its formation $\left(r<r_{\mathrm{cr}}\right)$, which could be provided by the micro heater through rapid pulse heating in the solution. This analysis is consistent with experimental observations that the input power required for incipient bubble generation increases with the DNA concentration because of a larger nucleation work required for that case. It is pertinent to note that a larger input power to the heater will lead to a higher bubble nucleation temperature from the heat transfer point of view. This will in turn lower the nucleation work to a certain extent (figure 9) to facilitate the nucleus formation.

4.2.2. Bubble dynamics in DNA solution. The existence of ssDNA macromolecules can affect not only the input power required for incipient bubble nucleation, but also greatly affect the evolution of bubble dynamics in the solution. Generally, the moving fluid in a polymer solution will carry polymer molecules at each location with the same fluid velocity at that location. However, this movement will be opposed by the cohesive force between the polymer molecules. Furthermore, the interactions of polymer molecules will in turn perturb the fluid flow. Thus, polymer chains (figure $7(b)$ ) are considered to be the centers of the resistance to fluid flow. In essence, this kind of hydrodynamic intermolecular interactions in the polymer solution will increase the flow impedance, leading to an increase of the solution's viscosity. Even more complex rheological phenomenon may also be induced, such as the non-Newtonian and viscoelasticity behavior of the fluid. In the following analysis, we will examine the effect of ssDNA macromolecules in a dilute solution on the viscosity increase in the solution.

Basically, when the polymer concentration is below the overlap concentration, viscoelastic and entanglement effects of the fluid are negligible [21]. In other words, polymer solutions are purely viscous in this case. As the concentration of the solution is increased, the viscosity will be dominated by intermolecular interactions of polymer macromolecules with increasing order. In dilute solutions, the polymer dynamics can be described by Zimm's model [22]. Accordingly, the relaxation time of the ssDNA macromolecule in theta solvent is given by $[22,23]$

$$
\tau=0.325 \frac{\eta_{\mathrm{s}} \bar{R}^{3}}{K_{\mathrm{B}} T}
$$

where

$$
\bar{R}=\sqrt{2 \xi_{\mathrm{p}} L_{\mathrm{c}}}
$$


Table 1. Total viscosity of the ssDNA solution at different concentrations. The solvent viscosity is $8.58 \times 10^{-4} \mathrm{~kg} / \mathrm{ms}$, and the relaxation time and intrinsic viscosity of the 60-base ssDNA are $4.7 \times 10^{-8} \mathrm{~s}$ and $1.6232 \times 10^{-2} \mathrm{~m}^{3} / \mathrm{kg}$ respectively.

\begin{tabular}{llll}
\hline Sample & $C\left(\mu \mathrm{g} \mu \mathrm{l}^{-1}\right)$ & $\eta \times 10^{4}(\mathrm{~kg} / \mathrm{ms})$ & {$[\eta] C$} \\
\hline ssDNA & 0.4 & 8.63 & 0.006492 \\
& 1 & 8.72 & 0.016232 \\
& 6.4 & 9.47 & 0.10388 \\
& 10 & 9.97 & 0.16232 \\
\hline
\end{tabular}

and the intrinsic viscosity of the ssDNA [22]:

$$
[\eta]=0.425 \frac{N_{\mathrm{A}}}{M} \bar{R}^{3}=1.308 \frac{R_{\mathrm{M}} T}{M \eta_{\mathrm{s}}} \tau .
$$

Note that both the relaxation time and intrinsic viscosity of the ssDNA are determined by the size and shape of individual ssDNA macromolecules, independent of its concentration. Taking into account the addition of ssDNA in the solvent, the total viscosity of the solution can be expressed as [24]

$$
\eta=\eta_{\mathrm{s}}(1+[\eta] C)
$$

The relaxation time, intrinsic viscosity of the ssDNA and the total viscosity of the DNA solutions can be calculated based on equations (12)-(15). The results are listed in table 1 , in which the last column gives the products of the intrinsic viscosity and concentration of the ssDNA. Since the products are all less than 1 , the ssDNA solutions used in the experiment can be treated as dilute solutions [21], which ensures the applicability of Zimm's model. It is shown from table 1 that the increase of the ssDNA concentration will lead to the increase of the total viscosity of the solution. The viscosity of $0.4 \mu \mathrm{g} \mu \mathrm{l}^{-1}$ ssDNA solution has a $0.6 \%$ increase compared with that of water. Similarly, the corresponding increase in viscosity of the ssDNA solution with concentrations of $1 \mu \mathrm{g} \mu \mathrm{l}^{-1}, 6.4 \mu \mathrm{g} \mu \mathrm{l}^{-1}$ and $10 \mu \mathrm{g} \mu \mathrm{l}^{-1}$ are $1.6 \%, 10.4 \%$ and $16.2 \%$, respectively. As mentioned previously, the increase of the viscosity of DNA solutions implies the increase of the flow impedance. This effect becomes very prominent at high concentrations, owing to larger hydrodynamic interactions of ssDNA macromolecules.

Basically, the vapor bubble growth process can be roughly divided into two stages: an isothermal process at the initial stage and a subsequent isobaric process [25]. For the isothermal process, the bubble growth is driven by the pressure difference between the inside bubble pressure (supposed to be at the saturation vapor pressure corresponding to the nucleation temperature) and the outside ambient pressure. For the isobaric process, the bubble growth is governed by the rate at which heat can be supplied from the superheated liquid layer to the bubble interface to facilitate the vaporization process. The bubble grows faster at the pressure driven stage, because the bubble radius is proportional to time; while at the thermal driven stage, it is proportional to the square root of time [25].

Generally, a source flow with a high velocity will be induced in the DNA solution at the initial stage of bubble growth [8]. During the bubble growth period, the bubble expansion work, which is consumed to overcome the surface tension and viscous dissipation (due to the flow of the liquid), has to be compensated by the internal energy of the vapor inside the bubble. This will lead to the decrease of the pressure inside the bubble although heat transferred from the heater to the bubble will increase the vapor pressure slightly at the same time. The net decrease in vapor pressure inside the bubble in turn will retard the bubble growth process due to the reduced driving force. The existence of ssDNA macromolecules in the solution will result in larger pressure drop in the bubble when the bubble grows. This is because of the increased viscous dissipation in this situation, due to the additional frictional forces exerted by the dissolved ssDNA macromolecules through hydrodynamic intermolecular interactions. As shown in figure $5(b)$, the bubble diameter at $0.5 \mathrm{~ms}$ (the first data point of each curve taken by the CCD) decreases with the increase of the ssDNA concentration, indicating the retardation effect on bubble growth.

From figure 5(b), we also find that for the cases of high DNA concentrations (6.4 and $10 \mu \mathrm{g} \mu \mathrm{l}^{-1}$ ), the bubble grows for about only $0.5 \mathrm{~ms}$ and then collapses, while the pulse heating process lasts for $1.6 \mathrm{~ms}$. This means that the bubble growth stopped even though the heating from the heater was still going on. Note that this phenomenon is quite different from the bubble growth process in DI water and low DNA concentrations, where the bubble growth period was comparable to the heating pulse widths. The reason for this unique phenomenon is that when a very large amount of ssDNA exists in the solution (high concentrations), the effects of viscous dissipation as bubble rapidly grows can be so serious that the vapor pressure inside the bubble quickly drops to lower than the outside ambient pressure even though the heating from the heater is still going on during this period. Under this situation, although the bubble can still grow due to inertia, it will quickly stop growing and begin to collapse because of the inversed pressure difference. This unusual phenomenon also implies that the heat transfer process from the micro heater to the bubble becomes ineffective because the heat transferred to the vapor bubble is unable to compensate for the pressure drop due to expansion work. A possible reason is that the existence of large number of ssDNA macromolecules in the aqueous solution can increase the thermal resistance for the heat transfer process due to their different thermal properties (i.e. conductivity and heat capacity) from those of water. It is also shown in figure $5(b)$ that a sudden collapse of the bubble occurred as the pulse heating was stopped. After the sudden collapse, the bubble pressure would be equal to the ambient pressure, and the subsequent collapsing process is dominated by the vapor condensation process.

In summary, the high concentrations of the DNA macromolecules in a solution can effectively retard the bubble growth by increasing the dissipation of bubble kinetic energy, and lowering the efficiency of thermal energy transport from heater to the bubble. As a result, both the bubble growth period and the recorded maximum bubble diameter are distinctively reduced at high DNA concentrations, as shown in figure 5(b). For medium and low concentrations ( 1 and $0.4 \mu \mathrm{g} \mu \mathrm{l}^{-1}$ ), the bubble growth period is comparable to the heating pulse width. Continuing growth of the bubble after heating was observed in $0.4 \mu \mathrm{g} \mu \mathrm{l}^{-1}$ ssDNA solution and DI water, which was due to the inertia effect as we observed in a previous study [8].

The bubble collapse process is intrinsically a relatively slow thermal controlled process, where the vapor bubble 
releases latent heat to the surrounding liquid and condenses on the heater surface until the bubble disappears. When a single bubble collapses, a sink flow will be induced in the fluid where fluid flows towards the center of the bubble [8]. As shown in figure 5(a), it took about $16 \mathrm{~ms}$ for the bubble's diameter to decrease from $1 \mu \mathrm{m}$ to zero in ssDNA solution with high concentrations ( 6.4 and $10 \mu \mathrm{g} \mu \mathrm{l}^{-1}$ ) during the bubble collapse process. However, the corresponding time at medium and low concentrations ( 1 and $0.4 \mu \mathrm{g} \mu \mathrm{l}^{-1}$ ) and in DI water was about $10 \mathrm{~ms}$. This means that the bubble collapse process is retarded at high DNA concentrations due to increased viscosities, as shown in table 1. Note that the distinctive shortening of the bubble lifetime at high concentrations is due to two factors: First, in these cases the bubble started to shrink even though the heating pulse was still on. Second, the bubble started to shrink at a smaller size compared with that at low concentrations (figure 5(b)).

\section{Concluding remarks}

In this paper, we have investigated a single micro vapor bubble generation in ssDNA solutions by a micro bubble actuator, and the bubble dynamics under various ssDNA concentrations and pulse widths. A distinct retardation effect on the motion of the micro bubble was observed at high ssDNA viscosities, due to the dissipation effect of the ssDNA macromolecules on bubble kinetics. It was observed that the bubble growth period was distinctively shortened at high ssDNA concentrations, leading to a reduced maximum bubble diameter and bubble lifetime. Based on classical thermodynamics and a simple model for nucleus formation in ssDNA solution, an analysis reveals that the bubble nucleation work will increase with the increase in DNA concentrations, which is consistent with experimental observations. The results of this investigation provide more information about the micro bubble generation process in DNA solution, which has been extensively employed in DNA transport based on micro thermal ink jet printer technology.

\section{Acknowledgments}

The authors would like to thank Enoch Kwok of Kishimoto Sangyo (HK) Co., Ltd for providing free parylene samples (diX-C and diX-N). This work was supported by the Hong Kong Research Grant Council through RGC grant HKUST6014/0E2, and partially supported by HKUST Institute of Integrated Microsystem Grant HKUST I2MS01/02.EG08.

\section{References}

[1] Tsai J and Lin L 2002 Active microfluidic mixer and gas bubble filter driven by thermal bubble micropump Sensors Actuators A 97-98 665-71
[2] Takahashi K, Yoshino K, Hatano S and Nagayama K 2001 Novel application of thermally controlled micro bubble driving system Micro Electro Mechanical Systems MEMS 2001: The 14th IEEE Int. Conf. pp 286-9

[3] Lin L 1998 Microscale thermal bubble formation: thermophysical phenomena and applications Microscale Thermophys. Eng. 2 71-85

[4] Yang W J and Tsutsui K 2000 Overview of boiling on microstructures-macro bubbles from micro heater Microscale Thermophys. Eng. 4 7-24

[5] Avedisian C T, Osborne W S, Mcleod F D and Curley C M 1999 Measuring bubble nucleation temperature on the surface of a rapidly heated thermal ink-jet heater immersed in a pool of water Proc. R. Soc. Lond. A 455 3875-99

[6] Deng P G, Lee Y K and Cheng P 2003 The growth and collapse of a micro-bubble under pulse heating Int. J. Heat Mass Transf. 46 4041-50

[7] Deng P G, Lee Y K and Cheng P 2003 Design and characterization of a micro single bubble actuator Transducer'03: the 12th Intl. Conf. on Solid-State Sensors, Actuators and Microsystems (Boston June 8-12) pp 647-50

[8] Okamoto T, Suzuki T and Yamamoto N 2000 Microarray fabrication with covalent attachment of DNA using bubble jet technology Nat. Biotechnol. 18 438-41

[9] Shima A and Tsujino T 1976 The behavior of bubbles in polymer solutions Chem. Eng. Sci. 31 863-9

[10] Brujan E A 1993 The effect of polymer additives on the bubble behavior and impulse pressure Chem. Eng. Sci. 48 3519-27

[11] Tanasawa I and Yang W J 1970 Dynamic behavior of a gas bubble in viscoelastic liquids $J$. Appl. Phys. 41 4526-31

[12] Plesset M and Prosperetti A 1977 Bubble dynamics and cavitation Annu. Rev. Fluid Mech. 9 145-85

[13] Schmidt E M 1983 Parylene as an electrode insulator-a review J. Electrophysiol. Technol. 10 19-29

[14] Frenkel J 1955 Kinetic Theory of Liquids (New York: Dover)

[15] Cole R 1974 Boiling nucleation Advances in Heat Transfer vol 10 (New York: Academic)

[16] Blander M and Katz J 1975 Bubble nucleation in liquids AIChE J. 21 833-46

[17] Kashchiev D 1982 On the relation between nucleation work, nucleus size, and nucleation rate J. Chem. Phys. 76 5098-102

[18] Skripov V P 1974 Metastable Liquids (New York: Wiley)

[19] Volkov A G, David W D, Darrell L T and Vladislav S M 1998 Liquid Interfaces in Chemistry and Biology (New York: Wiley)

[20] Cantor C R and Schimmel P R 1980 Biophysical Chemistry Part III The Behavior of Biological Macromolecules (San Francisco: W H Freeman)

[21] Bohdanecky M and Kovar J 1982 Viscosity of Polymer Solutions (Amsterdam: Elsevier)

[22] Doi M and Edwards S F 1986 The Theory of Polymer Dynamics (Oxford: Clarendon)

[23] David B 2002 Mechanics of the Cell Part I (Cambridge: Cambridge University Press)

[24] Cantor C R and Schimmel P R 1980 Biophysical Chemistry Part II Techniques for the Study of Biological Structure and Function (San Francisco: W H Freeman)

[25] Mikic B B, Rohsenow W M and Griffith P 1970 On bubble growth rates Int. J. Heat Mass Transf. 13 657-66 